\title{
The Inspiration of Large-scale Settlement of Modern Suburban Areas in the Neighborhood Center in Suzhou and Chuzhou Industrial Park
}

\author{
Rongyu SUN \\ Southwest Jiaotong University \\ Chengdu, China \\ e-mail: 771058647@qq.com
}

\author{
Jiayin GUO
Agriculture University of Hebei Province \\ Jiayin GUO
Agriculture University of Hebei Province \\ Baoding, China \\ e-mail: $170856121 @ q q . c o m$
}

\begin{abstract}
The development of Neighborhood Center in overseas and domestic is illustrated in this article under the background of current situation of large-scale settlement of modern suburban areas. Here, the 9 neighborhood centers in $\mathrm{Su}$ and $\mathrm{Chu}$ industrial park were investigated and analyzed in the first place, and then the relevant principles were drawn as: location in the suburban areas, service demand and the rationality of scope of service, the richness and specialty of functional commercial activities and the humanity in communication space.
\end{abstract}

Keywords-neighborhood center; Suzhou and Chuzhou industrial park; large-scale settlement

\section{INTRODUCTION}

On a general review of the current situation of our settlements in urban areas, there are always some problems like unban construction and lag public service. The demand of service for life needs to be satisfied in a long time by the market. And that makes it possible for the formation and development of the market in the street which causes the deterioration of the environment. Thus, the universal problems are the lack of public service, the faultiness of configuration in function, the weakening of community interaction, the closure of community and the chaos of the community surroundings.

\section{The DEVELOPMENT OF NeIGHBORHOOD CENTER}

\section{A. Neighborhood Center}

The phrase "Neighborhood Center" comes from Singapore and it is a new format originated from "Neighborhood Unit". It refers to the integrated service center of the community which occupies 12 functions included banks, supermarkets, postal services, restaurants, laundry rooms, hair salon, drug stores, stationery stores, maintenance places, activity centers, vegetable markets and community clinics that offers supporting services for residents in surrounding communities. Now, the neighborhood centers in Singapore have already formed perfect public matching systems which included regional center, town center, neighborhood center, residential precinct center and other matching utilities. (See figure 1)

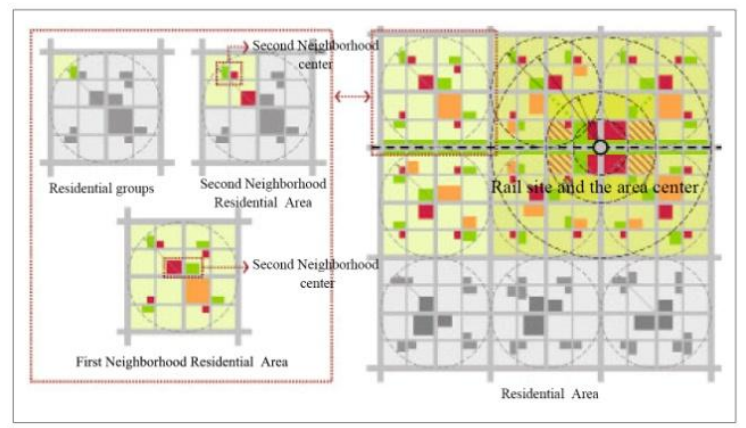

Figure 1. System of Planning Hierarchy in Neighborhood Center of Singapore (Zhuhai Construction Bureau of Urban and Rural Planning)

\section{B. Development of Neighborhood Center}

The domestic development of neighborhood center is on the basis of experiences from Singapore. Experiments were doing in Suzhou industrial park, Sino-Singapore Eco-City, Nanjing, Zhenjiang, Mingxin city in Shenzhen, Beijing, Zhuhai, Chuzhou city in Anhui and other cities, and the results were fantastic. The most obvious results are intensive use of land, better environment, good habitability, a comfortable and harmonious social space and better neighborhood communication. At the same time, it can enhance the density of living space, functions, and the quality of personal life as well shapes an abundant spatial arrangement.

The development of western neighborhood center can list as follows: the theory of working class district of Robert Owen, garden city of Ebenezer Howard, "Neighborhood Unit" of Clarence Perry (see figure 2), urbanism of Le Corbusier the architecture, urban theorist Jane Jacobs's book The Death and Life of Great American Cities, all of these have made great dedication. Among these theories, Perry's "Neighborhood Unit" influences a lot of the neighborhood center in Singapore which emphasizes the organized public life, and the intensive arrangement of commercial, medical and educational service facilities in order to make the daily life easier. However, large-scale medical and educational service facilities have less efficiency in residents' daily life. Therefore, it is enough to satisfy the radius scope of the service. It can be established in and the edge of the community on the occasion of the living specialty and the green belts. (See figure 3 ) 


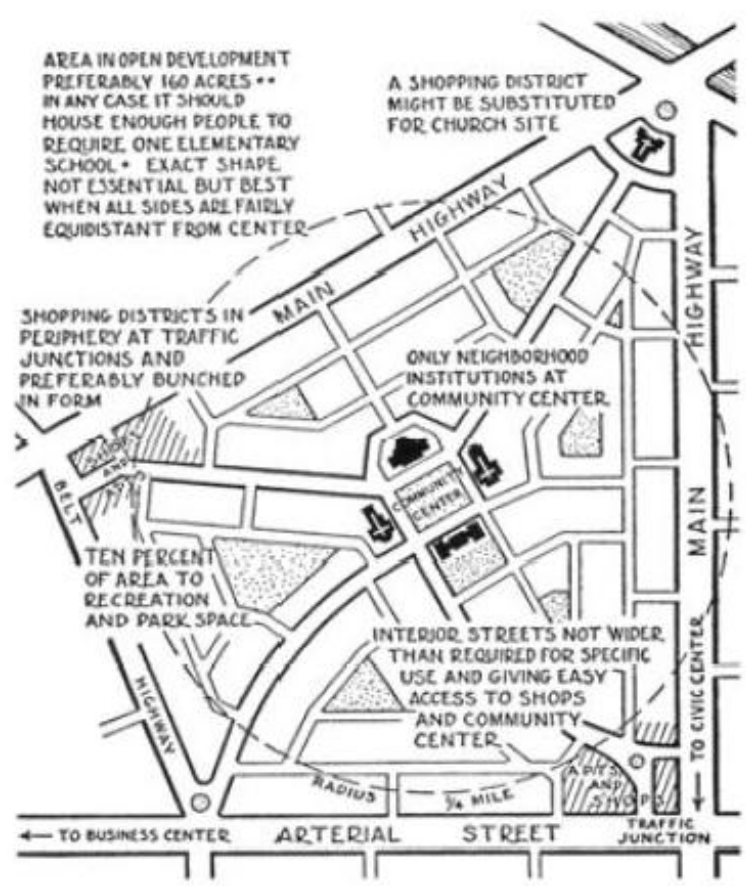

Figure 2. Model of Perry's Neighborhood Unit (originated from Duanyplater-Zyberk, E.2008:84)
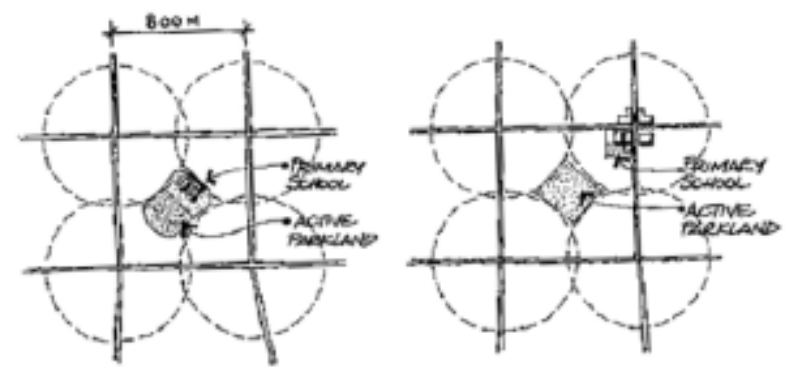

Figure 3. Spatial relationships of planned park, primary school and neighborhood center in Western Australia (originated from Western

Australia Planning Commission, Department for Planning and Infrastructure, 2009.)

\section{ANALYSIS OF NEIGHBORHOOD CENTER IN SUZHOU AND CHUZHOU INDUSTRIAL PARK}

\section{A. Features of Scale and Radius of Service}

\section{1) Scale of service:}

On the basis of the data above, it can be concluded that Neighborhood Center is a kind of service that surpasses market scale. There is a difference between Neighborhood Center and business center in the suburban areas, the former possesses the quality of openness and sharing. According to the differences, the 9 neighborhood center scan is divided into large, medium and small, see the table 1 below.

Compare to the different kinds of neighborhood centers, three of the small ones have the limitation of lower service quality. Among these: Gudu occupies lower percentage except educational facilities; Shihui and Qinyuan own worse service quality and fewer users.
TABLE I. Different Models in Neighborhood Center of Su and Chu (Originated from the Writer)

\begin{tabular}{|c|c|c|}
\hline $\begin{array}{c}\text { Scale and } \\
\text { type }\end{array}$ & Floor area & $\begin{array}{c}\text { Name of Neighborhood } \\
\text { Centers }\end{array}$ \\
\hline \multirow{3}{*}{ small } & \multirow{3}{*}{$\leq 1500 \mathrm{~m}^{2}$} & Shihui \\
\hline & & Qinyuan \\
\hline & & Guidu \\
\hline \multirow{3}{*}{ medium } & \multirow{3}{*}{$1500-2500 \mathrm{~m}^{2}$} & Dongsheng \\
\hline & & Xincheng \\
\hline & & Linglong \\
\hline \multirow{3}{*}{ large } & \multirow{3}{*}{$\geq 2500 \mathrm{~m}^{2}$} & Jinji \\
\hline & & Wanzhou \\
\hline & & Hudong \\
\hline
\end{tabular}

The medium and large ones such as Dongsheng in Chuzhou, Jinjihu in Suzhou, and Hudong, by contrast, are better in functions and quality. Conclusion can be drawn that the construction scale has to reach a certain to maintain the supply, and it acquires a higher demand of land.

2) Radius of service scope:

It was the standard of radius of 500 meter and 30,000 people as a unit when this work was established in Suzhou and Chuzhou on the experience of Singapore. In this kind of layout, a format of match between spatial scale and population standard which pay more attention to population and space, the double scale has proposed. Under this double scale, residential zone and residential quarters has become two-level neighborhood center. (See figure 4)

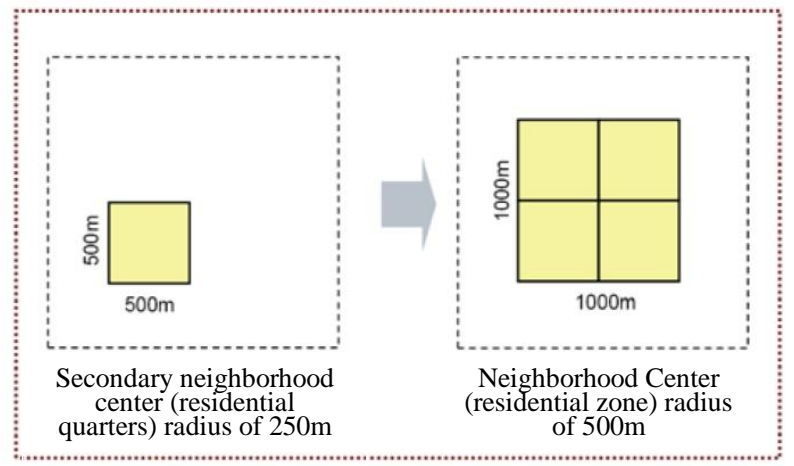

Figure 4. The structure of two-level nuclear unit in Neighborhood Center and secondary Neighborhood Center (originated from the writer)

\section{B. Functions and Commercial Activities}

\section{1) Integrated features of commercial activities:}

In the 9 neighborhood centers in research, all of them are equipped with the function of shopping, service, entertainment, catering and education and feature the integrity of neighborhood center in the format. The proportion of commerce is unusually under the control of 50\% except Jinjihu.

As community commerce, commerce and life service are still the most important parts. They occupy $60 \sim 70 \%$ in average among all the functions. Some of the centers highlight community service such as Guidu and it reaches 
53\%. (See figure 5)

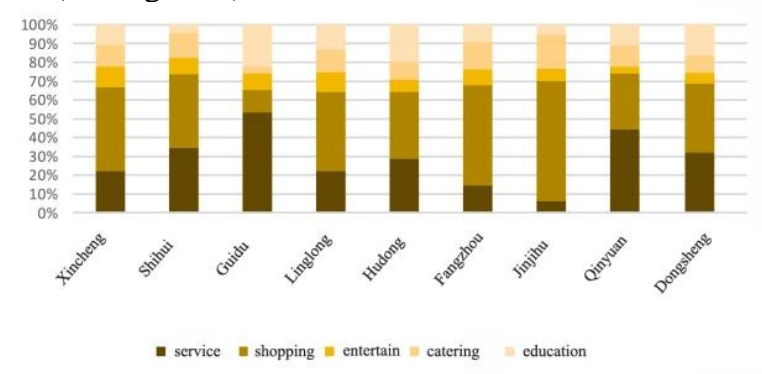

Figure 5. Form of commercial activities in $\mathrm{Su}$ and $\mathrm{Chu}$ Neighborhood Center (Originated from the writer)

\section{2) Characteristic features of commercial activities:}

As the main body of all the neighborhood centers in Suzhou, Suzhou Industrial Park Neighborhood Center Development Co., Ltd controls from a whole perspective. There are special settled service programs to show the characteristics in every neighborhood center include fresh food in neighborhood, community contact office and community health service station.

\section{Features of Neighborhood Communication}

There are many types of commercial activities in neighborhood center of Suzhou and Chuzhou, it provides all kinds of communication activities. According to the theory of Jan Gehl in his book Life between Buildings, all of the activities can be divided into 3 types: necessity activity, selectivity activity and social intercourse activity (see the table 2 below). Different activities lead to different intercourses, therefore, different kind of activities are the best premises to make better communication. The functions in the neighborhood center can be divided into shopping, service, catering, entertainment and education.

TABLE II. The Relevance of Communication Activities and the Quality of Environment

\begin{tabular}{|c|c|c|}
\hline \multirow{2}{*}{ Scale and type } & \multicolumn{2}{|c|}{ Quality of material environment } \\
\cline { 2 - 3 } Necessity activity & bad & good \\
\hline Selectivity activity & & \\
\hline $\begin{array}{c}\text { Social intercourse } \\
\text { activity }\end{array}$ & & \\
\hline
\end{tabular}

1) Features of shopping interaction:

Shopping belongs to the necessity activities which has the property of purpose in the outdoor activities with strong purpose and less human contact. Thus, the function of shopping is not the main function of communication activities. However, not any of them stand alone; they can transform to each other and coexist. For example, shopping sometimes transforms to catering, entertainment and service. This acquires higher spatial environment.

2) Features of entertainment and service:

The layout of service in neighborhood center of Suzhou and Chuzhou is nearly 25\%. It is not a fixed number and it is installed according to the population in community, such as in Guidu, this function is occupied more than $50 \%$. Entertainment and service are the main activities, and they are also the behaviors that stay longer and more frequent contact that provide better condition of communication.

\section{3) Features of catering:}

Residents in neighborhood center have little demand in catering. The main pack is the office works who work here that are served in snacks which covers little in the function of catering. It can be concluded that the activities in catering are not as strong as other functions and it is not the main communication activity.

\section{4) Features of education:}

Nowadays, people have paid more attention on education; there is a saying in China: Don't let the children fail at the first start. When doing the research, it came out that education is the most popular activities. Here, children, adults and old people can be found everywhere and the interactions get along well.

\section{INSPIRATIONS ON MODERN SUBURBAN AREAS} ENCOURAGED BY NEIGHBORHOOD CENTER OF SUZHOU AND CHUZHOU INDUSTRIAL PARK

As a new form of domestic community commerce, the development of neighborhood center is still at the first stage. The introduction of neighborhood center in Suzhou and Chuzhou industrial park provides a strong demonstration effect to the whole country. Through the research of features of neighborhood center and specialty in the Suzhou industrial park, the influence fits the problems of our suburban areas well. In the future, there is great potential of neighborhood center and it will be applied in more cities with the development of suburbanization and counter urbanization.

\section{A. Principle of Selecting Sites in Suburban Areas}

Considering the location and scale, it acquires an integrated neighborhood center for residents' daily life. It has the advantage of better utilization of land resources; more attractive of community; and at the same time, it avoids the repetition, waste and weakening of the community culture. Usually, it is the area that far away from downtown, flat and convenient in traffic that makes it easier for the exploitation and plan as the form of industrial parks.

\section{B. Principle of Scale and Radius of Service}

The scale and radius of service can be influenced by the unban level of development in different cities and residents'travel. In Suzhou industrial park, the interval is usually 1 kilometer, and it can be enlarged to 2 3 kilometers on the occasion of small or high-rank communities. Therefore, according to features of different areas, the radius of service is supposed to set on occasion with certain 
purposes. In the common communities, it is better to set the radius as 500 800 meters to make them walk there. In some special communities which include high-rank communities, common communities in a low density and well-developed public traffic utilities, the radius can be enlarged in a certain scale and less than 1500 meters.

\section{Principle of Richness and Characteristic of Commercial Activities and Local Conditions}

According to the domestic and oversea experience of neighborhood center, the integrality and characteristic should be considered into the function of modern suburban communities.

(1) Richness of features: the functions of neighborhood center should include bank, supermarket, postal service, catering, laundry room, hair solon, drug store, stationery store, maintenance, activity center, vegetable market, community clinic the 12 essentials to ensure the service level and satisfy residents.

(2) Characteristic service: the characteristic service of the community is supposed to put in an obvious position, for example, community committee, sub-district office and community health service station can be included in their work or they can remove the community primary units to the neighborhood center to offer the residents one-stop service.

(3) Local conditions: put the features of the local residents into consideration and at the same time, supply and adjust the differences of the commercial activities. For example, Qinyuan neighborhood center consider a lot for the need of Koreans about the existence of Korean enterprises.

\section{Principle of Humanity in the Communication Space}

The inner place of the neighborhood center needs to be more humanity to attract for residents' longings and staying to improve the blending of community culture, such as enhance the quality of space service and strengthen the usage of educational function. Meanwhile, the space of catering and shopping should be comfortable as well. Platforms and seats for leisure, especially half-private places are supposed to install to offer residents places to have a rest and conversation. The neighborhood center is the symbol of community and it represents the energy, quality and culture of one community.

\section{CONCLUSION}

In this article, detailed researches and summarization of the 9 neighborhood centers in Suzhou and Chuzhou industrial park were illustrated and the similarities and differences of different neighborhood centers were analyzed which hold lessons for the construction of large-scale suburban areas. Effective principles focus on the selection of sites, scale and radius of service, features of commercial activities and the setting of communication space are explored to help the plan and design of large-scale suburban neighborhood centers.

\section{REFERENCES}

1. Liu Quan, Zhang Zhenyu. Significance of Spatial Scale: Reflections on Public Facilities Planning in Residential Area under Neighborhood-centered Pattern in Zhuhai.

2. Shi Jianjie, Zhu Xiaofang, Maqiang. Plan and Research of Public Service Facilities in Community - Case study on Zhenjiang city Runzhou Quarter [C] // China City Planning Institute. Planning and Innovation: 2010 Annual Conference of Chinese Urban Planning. Chongqing: Chongqing Press.

3. Planning Bureau of Nanjing city. A Discussion of Direction of Public Facility Criteria of New Community in Nanjing (draft version) [S]. Nanjing: Planning bureau of Nanjing city, 2004.

4. Wu Nan. Study of the Layout of Ecology-oriented Community Center-Case study on Sino-Singapore Eco-City [C] // China City Planning Institute. Transformation and reconstruction: Essays on the annual conference of the 2011 China Urban Planning. Nanjing: Dongnan University Press.

5. [Denmark] Jan Gehl. Life between Buildings Beijing: China Construction Industry Press, 2002. 